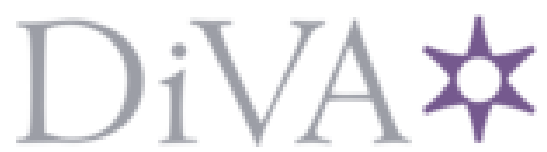

http://www.diva-portal.org

This is the published version of a paper published in American Journal of Obstetrics and Gynecology.

Citation for the original published paper (version of record):

Collins, E., Strandell, A., Granåsen, G., Idahl, A. (2019)

Menopausal symptoms and surgical complications after opportunistic bilateral salpingectomy, a register-based cohort study

American Journal of Obstetrics and Gynecology, 220(1): 85.e1-e10

https://doi.org/10.1016/j.ajog.2018.10.016

Access to the published version may require subscription.

N.B. When citing this work, cite the original published paper.

Permanent link to this version:

http://urn.kb.se/resolve?urn=urn:nbn:se:umu:diva-155211 


\title{
Menopausal symptoms and surgical complications after opportunistic bilateral salpingectomy, a register-based cohort study
}

\author{
Elin Collins, MD; Annika Strandell, MD, PhD; Gabriel Granåsen, MSc; Annika Idahl, MD, PhD
}

BACKGROUND: In recent years, the fallopian tubes have been found to play a critical role in the pathogenesis of ovarian cancer. Therefore, bilateral salpingectomy at the time of hysterectomy has been proposed as a preventive procedure, but with scarce scientific evidence to support the efficiency and safety.

OBJECTIVE: Our primary objective was to evaluate the risk of surgical complications and menopausal symptoms when performing bilateral salpingectomy in addition to benign hysterectomy. Furthermore, we sought to compare time in surgery, perioperative blood loss/blood transfusion, duration of hospital stay, days to normal activities of daily living, and days out of work for hysterectomy with bilateral salpingectomy compared with hysterectomy only. A secondary objective was to study the uptake of opportunistic salpingectomy in Sweden.

STUDY DESIGN: This was a retrospective observational cohort study based on data from the National Quality Register of Gynecological Surgery in Sweden. Women $<55$ years of age who had a hysterectomy for benign indications with or without bilateral salpingectomy in 1998 through 2016 were included. Possible confounding was adjusted for in multivariable regression models.

RESULTS: During the study period, 23,369 women had a hysterectomy for benign indications. The frequency of bilateral salpingectomy at the time of hysterectomy increased mainly from 2013, which is why the period
2013 through mid-2016 was selected for further analysis $(n=6892)$. There was a low frequency of vaginal hysterectomy with bilateral salpingectomy performed in this period, which is why only abdominal and laparoscopic surgeries were selected for comparative analysis $(n=4906)$. This study indicates an increased risk of menopausal symptoms (adjusted relative risk, 1.33; 95\% confidence interval, 1.04-1.69) 1 year after hysterectomy with bilateral salpingectomy compared with hysterectomy only. Hospital stay was 0.1 days longer in women having salpingectomy $(P=.01)$, and bleeding was slightly reduced in the salpingectomy group $(-20 \mathrm{~mL}, P=.04)$. Other outcome measures were not significantly associated with salpingectomy, albeit a tendency toward higher risk of minor complications was seen (adjusted relative risk, 1.30; 95\% confidence interval, 0.93-1.83).

CONCLUSION: Bilateral salpingectomy at the time of hysterectomy was associated with an increased risk of menopausal symptoms 1 year after surgery. Randomized clinical trials reducing the risk of residual and unmeasured confounding and longer follow-up are needed to correctly inform women on the risks and benefits of opportunistic salpingectomy.

Key words: complications, hysterectomy, menopausal symptoms, opportunistic salpingectomy

\section{Introduction}

Ovarian cancer is a considerable cause of morbidity and mortality around the world, with approximately 239,000 new cases and 159,000 deaths each year. ${ }^{1}$ In recent years a new theory has been put forward where the fallopian tubes play a critical role in the pathogenesis of certain types of ovarian cancer. ${ }^{2,3}$ Retrospective observational register-based studies from Denmark and Sweden have found a decreased incidence of ovarian cancer in

Cite this article as: Collins $E$, Strandell A, Granåsen G, et al. Menopausal symptoms and surgical complications after opportunistic bilateral salpingectomy, a registerbased cohort study. Am J Obstet Gynecol 2019;220:85.e1-10.

\section{2-9378}

(c) 2018 The Author(s). Published by Elsevier Inc. This is an open access article under the CC BY-NC-ND license (http:// creativecommons.org/licenses/by-nc-nd/4.0/). https://doi.org/10.1016/j.ajog.2018.10.016 women who have had salpingectomy compared with women who have not undergone surgery. ${ }^{4,5}$ These findings have led to an increasing number of opportunistic salpingectomies as a preventive measure for ovarian cancer when performing a hysterectomy for benign reasons. $^{6-8}$ Some regions and/or national societies recommend opportunistic salpingectomy to be considered, both in Europe ${ }^{9}$ and in North America. ${ }^{10,11}$ This, despite the lack of scientific evidence in regards to the safety of performing opportunistic salpingectomy in addition to hysterectomy. Furthermore, hysterectomy by itself confers a reduced risk of ovarian cancer ${ }^{12}$ and an additional risk-reducing effect of salpingectomy is not proven.

In 2017 there were 2 systematic reviews published, ${ }^{13,14}$ identifying 11 studies that compared hysterectomy with hysterectomy and simultaneous bilateral salpingectomy regarding perioperative data, complications, and/or effect on ovarian function. ${ }^{6,8,15-23}$ The included studies were either small or had a short follow-up, which applies to the few studies published thereafter as well. $^{7,24-26}$ No significant negative effects have been shown from having the salpinx removed at the time of hysterectomy, aside from 2 studies showing 12 and 16 minutes longer duration of surgery, respectively.

Studies analyzing the potential effect on ovarian function are small and use surrogate measures such as anti-müllerian hormone $(\mathrm{AMH})$, follicle stimulating hormone (FSH), estradiol, and sonographic imaging. ${ }^{15,20,23-25}$ To our knowledge, no published study has analyzed subjective symptoms of menopause in the women.

Our primary aim was to analyze menopausal symptoms, complication 


\section{AJOG at a Glance}

Why was this study conducted?

We conducted this study as a response to the increasing frequency of opportunistic salpingectomy at the time of hysterectomy in many parts of the world, despite a lack of evidence that it can be done without harm.

\section{Key findings}

In a national register-based analysis of women subjected to hysterectomy with bilateral salpingectomy compared with hysterectomy only, an association with increased risk of menopausal symptoms 1 year after opportunistic salpingectomy was found.

\section{What does this add to what is known?}

The possibility that salpingectomy might affect ovarian function is a key consideration in the decision to perform opportunistic salpingectomy. No prior study focuses on menopausal symptoms as a consequence of opportunistic salpingectomy; as such, this is an important addition to the discussion.

rate, and perioperative data after hysterectomy with bilateral salpingectomy compared with hysterectomy only in a national register-based cohort study. A secondary aim was to assess changes over time in the uptake of opportunistic salpingectomy in the years 1998 through mid-2016 in Sweden.

\section{Materials and Methods}

A retrospective observational cohort study, based on the Swedish National Quality Register of Gynecological Surgery $(\mathrm{GynOp})^{27}$ was conducted. Data were analyzed on the GynOp server with no access to personal identification of the subjects. Ethical approval was obtained from the regional ethical review board, University of Gothenburg, Sweden, Oct. 24, 2016 [Dnr T945-16 (50116)].

Women age $<55$ years who had a hysterectomy for benign reasons with or without bilateral salpingectomy 1998 through mid-2016 in Sweden and had their surgery registered in GynOp were included. The register started in 1997 and has gradually increased the number of reporting clinics. At the end of the study period about $75 \%$ of gynecological clinics performing surgery in Sweden reported to the register and approximately $90 \%$ of hysterectomies performed in these clinics were included. 28,29
The register collects data directly from the patients through validated questionnaires preoperatively, and at 8 weeks and 1 year postoperatively. All questionnaires are assessed by the physician responsible for the surgery. ${ }^{30}$ The gynecologist registers medical information in GynOp upon decision to perform surgery, at surgery, at discharge from hospital, and when assessing the postoperative questionnaires from the patients. ${ }^{30-32}$ Reports of complications are registered at discharge and at 8 weeks and 1 year postoperatively. The complications are graded as minor or severe and subdivided into type and/or location, ie, damage to the ureter, lower urinary tract, vagina, intestines, abdominal wall, nerve damage, fistula formation, and pain. ${ }^{31}$

Menopausal symptoms are registered by the patients in questionnaires preoperatively and at 1 year postoperatively. Preoperatively, the question reads, "Do you have, or have you had, menopausal symptoms (flushes, sweats, palpitations)?"; whereas the postoperative question reads, "Do you have menopausal symptoms (flushes, sweats, palpitations)?" Women also answered the question "Do you use hormonal supplements with estrogen?" both preoperatively and postoperatively, where only the answer "Yes-for menopausal symptoms" was considered as treatment for menopausal symptoms in the analysis. Other options were "Yes—for vaginal problems," "Yes_for urinary tract problems," and "Yes-other reason." 27

We analyzed data on outcomes up to 1 year postoperatively. The outcomes duration of surgery, perioperative bleeding, administered units of blood, length of hospital stay, days out of work, and days to normal activities of daily living (ADL) were registered during hospital stay and/or at 8 weeks postoperatively. Outcomes collected at the 1-year follow-up were menopausal symptoms and complications.

Based on the trend with increasing rates of bilateral salpingectomy at benign hysterectomy in Sweden from 2013 (Figure 1), the years 2013 through mid2016 were selected for the analyses regarding outcomes up to 8 weeks after surgery. To have near complete data from the 1-year follow-up regarding complications and menopausal symptoms, the cohort having surgery in 2013 through March 2015 was analyzed. It was not possible to assess the prevalence of benign pathologies in the fallopian tubes, ie, hydrosalpinx, due to the design of the register.

Exclusion criteria were simultaneous surgery on the ovaries, perioperative findings suggesting malignancy, or anatomical pathology with malignant/ premalignant findings.

For comparison between 2 groups Student $t$ test or Mann-Whitney $U$ test were used for continuous variables; for categorical variables $\chi^{2}$ test or Fisher exact test where applicable were used to assess proportions. All statistical tests were 2 -sided and a $P$ value $<.05$ was considered to indicate statistical significance. Possible confounding was adjusted for in multivariable regression models. Relative risk for binary outcomes were calculated with Poisson regression models and continuous outcomes using linear regression. The choice of possible confounders was guided through directed acyclic graphs, clinical relevance, and the change-inestimate criterion $(>10 \%)$.

An age-stratified analysis $(<40$, $40-44,45-49,50-54$ years) regarding the outcome "menopausal symptoms" was performed and the regression 


\section{FIGURE 1}

\section{Selection process of analyzed cohort}

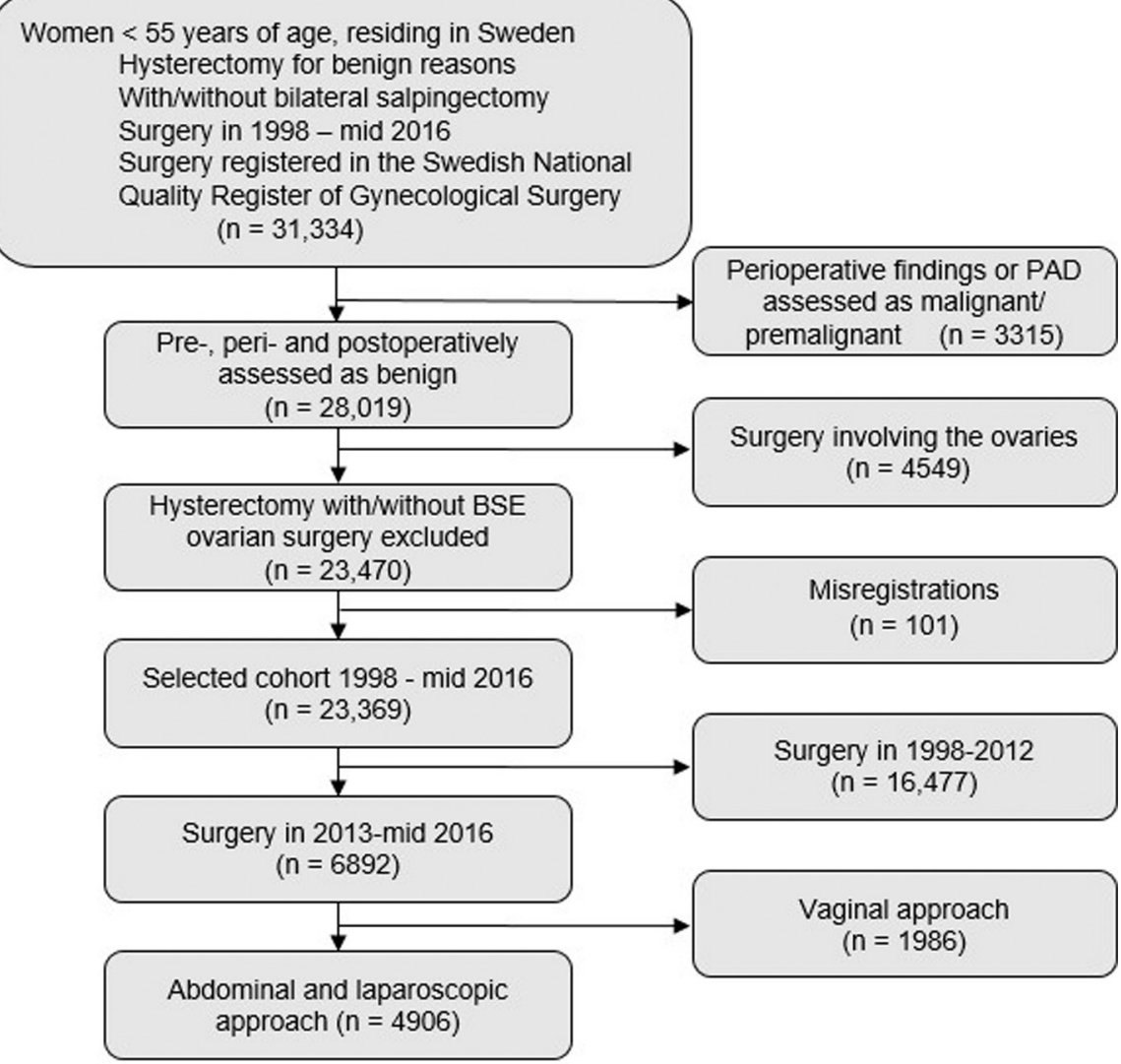

Flow diagram with selection process of cases for analysis, including reasons for exclusion. $B S E$, bilateral salpingectomy; $P A D$, pathologic-anatomic diagnosis.

Collins et al. Complications after opportunistic salpingectomy. Am J Obstet Gynecol 2019.

analysis included the same variables as in non-age-stratified analysis except for age. Interaction between age and performed surgery (hysterectomy vs hysterectomy with bilateral salpingectomy) was tested in regard to the risk of developing menopausal symptoms.

Software (SPSS, Version 24.0; IBM Corp, Armonk, NY) was used for the statistical analysis.

\section{Results}

After the inclusion and exclusion process, 23,369 hysterectomies with or without bilateral salpingectomy from 1998 through the end of June 2016 were included in the analysis (Figure 1). From 2013 there was a rapid increase in the frequency of bilateral salpingectomy at the time of benign hysterectomy: $1.9 \%$ in $2012,8.9 \%$ in 2013 , to $37.8 \%$ in 2016 (Figure 2). Based on this, hysterectomies performed in 2013 through 2016 were chosen for further analysis. There were 6892 hysterectomies with or without bilateral salpingectomy registered in GynOp during this period and the surgical approaches were laparoscopic, abdominal, and vaginal.

In 2013 through mid-2016, 50.1\% of hysterectomies were carried out by the abdominal approach, $21.1 \%$ laparoscopically, and the remaining $28.8 \%$ vaginally. We found an increase in the proportion of bilateral salpingectomy in all surgical approaches, with the largest proportion in laparoscopic surgery (laparoscopic 63\%; abdominal 36\%; vaginal $4.4 \%$ ) (Figure 3). Only 42 vaginal hysterectomies with bilateral salpingectomy were registered in GynOp during this time period. Thus, only surgeries by laparoscopic and abdominal approach were included for further analysis $(\mathrm{n}=4906)$.

The basic characteristics of women in the 2 groups are described in Table 1 . Women who had bilateral salpingectomy were slightly older compared with women who had hysterectomy only (mean 45.1 years [interquartile range $\{\mathrm{IQR}\} 42-49$ years] vs 44.1 years [IQR 41-48 years], $P<.001)$. Furthermore, those having salpingectomy had more pregnancies (median 3.0 [IQR 2-4] vs 2.0 [IQR 2-4], $P<.001)$ and higher parity (median 2.0 [IQR 1-3] vs 2.0 [IQR 1-3], $P<.001)$. There were no significant differences in distribution of American Society of Anesthesiologists (ASA) classification (physical status), body mass index (BMI), smoking, previous abdominal surgery, and endometriosis, but there was a slightly higher percentage of women with previous salpingitis in the salpingectomy group $(8.1 \%$ vs $6.0 \%, P=.03)$.

Analyses of perioperative outcomes and complications up to 8 weeks are presented in Table 2. In adjusted analyses we found a slightly longer hospital stay after bilateral salpingectomy in combination with hysterectomy (0.1 day; $95 \%$ confidence interval [CI], 0.02-0.18), which equals 2 hours and 24 minutes. There was also a small but significantly reduced blood loss associated with bilateral salpingectomy when adjusted for surgical approach, smoking, BMI, endometriosis, previous salpingitis, and ASA classification $(-20 \mathrm{~mL} ; 95 \% \mathrm{CI}$, -40 to -0.1$)$. No significant differences in duration of surgery, administered units of blood, time to normal ADL, and days out of work were found. Furthermore, we found no difference in complications at discharge or after 8 weeks.

At the 1-year follow-up there was a significantly larger proportion of minor complications in the group subjected to bilateral salpingectomy (adjusted relative risk [aRR], 1.35; 95\% CI, 1.01-1.83) if adjusted for surgical route, BMI, and smoking status. If previous salpingitis was added to the model, the results were no longer significant $(\mathrm{aRR}, 1.30$; $95 \% \mathrm{CI}$, 
FIGURE 2

Uptake of bilateral salpingectomy at benign hysterectomy in Sweden

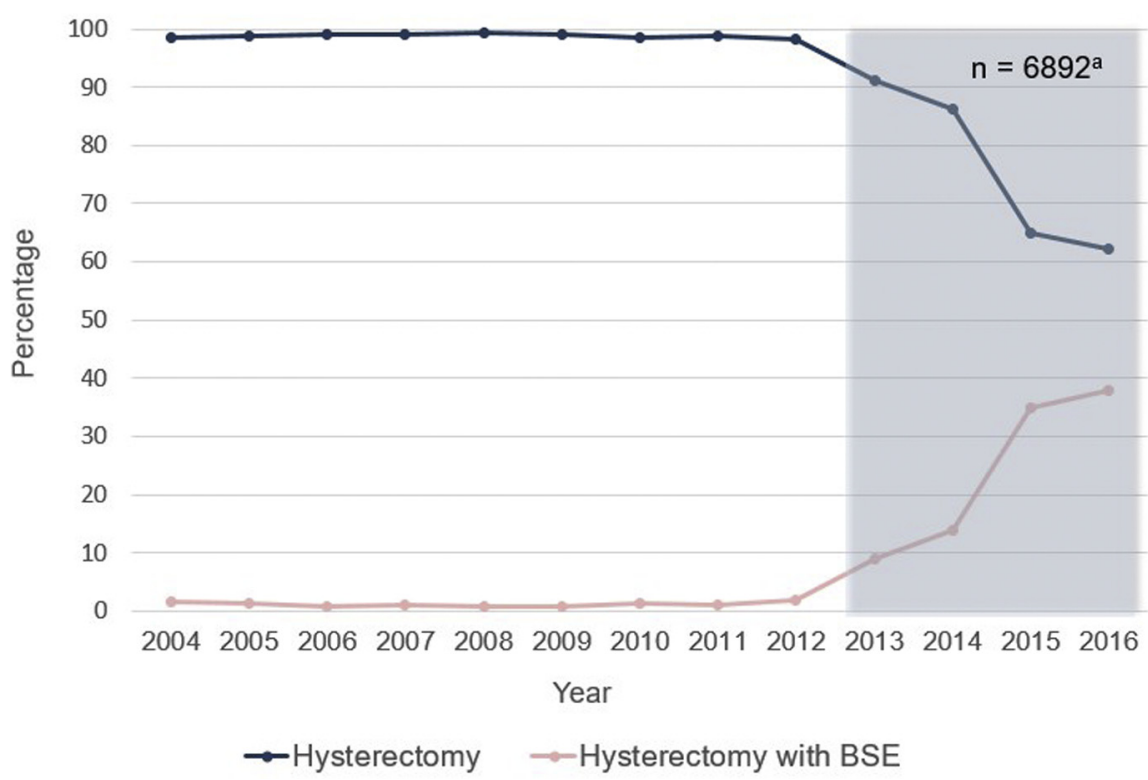

Rate of hysterectomy and hysterectomy with bilateral salpingectomy (BSE) for benign indications in Sweden 2004 through 2016. " Number of surgeries performed in 2013 through mid-2016; comparative analyses based on these cases.

Collins et al. Complications after opportunistic salpingectomy. Am J Obstet Gynecol 2019.

\section{FIGURE 3}

\section{Uptake of bilateral salpingecomy per surgical approach in Sweden}

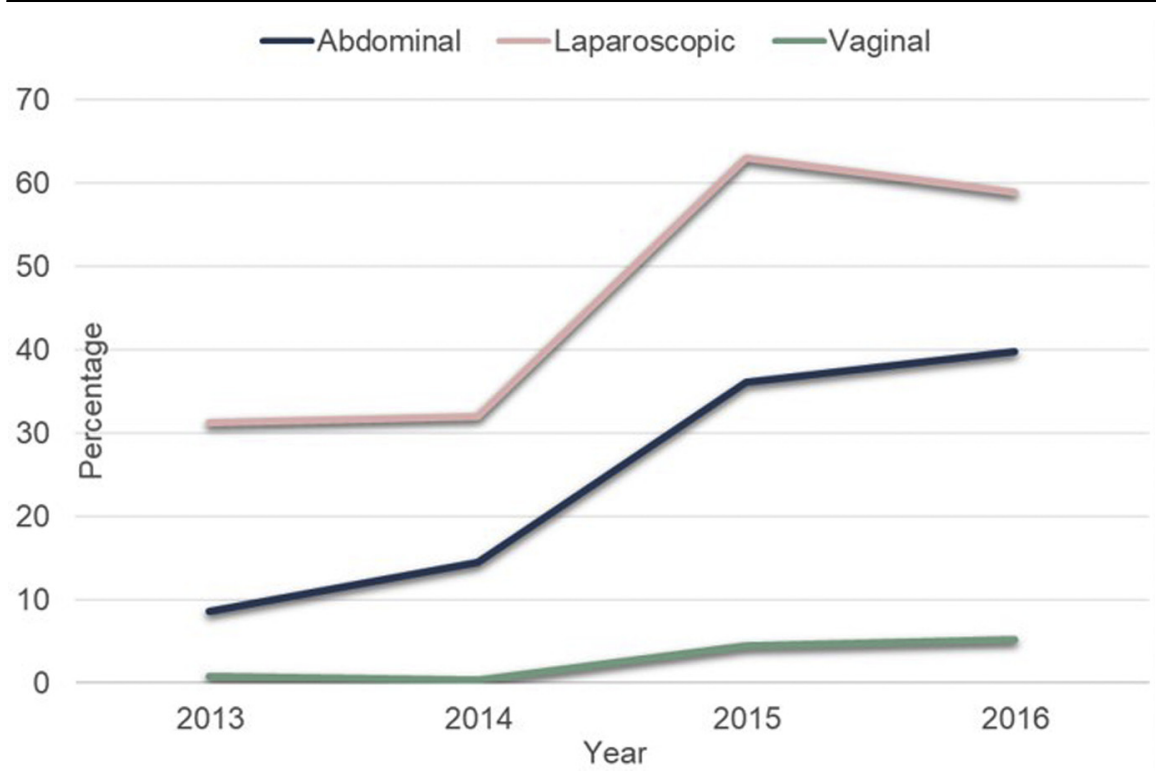

Proportion of benign hysterectomies where bilateral salpingectomy was carried out, per surgical approach (\%).

Collins et al. Complications after opportunistic salpingectomy. Am J Obstet Gynecol 2019.
$0.93-1.83)$. There were no differences in reported severe complications overall (aRR, 1.08; 95\% CI, 0.51-2.27) (Table 3).

Subdivided into type and degree of complication, only severe damage to the ureters was significantly increased after salpingectomy (relative risk [RR], 9.84; 95\% CI, 1.91-50.6). All cases of ureter damage were seen in patients having total hysterectomy. The increased risk associated with bilateral salpingectomy in addition to hysterectomy prevailed including only women with total hysterectomy (RR, 3.27; 95\% CI, 2.03-5.28) (Table 4).

The assessment of menopausal symptoms in our study is based on questionnaires preoperatively and 1 year postoperatively. There was no significant difference in reported menopausal symptoms between the 2 groups before surgery, but 1 year postoperatively there was a significantly increased risk of menopausal symptoms after bilateral salpingectomy compared with hysterectomy only (RR, $1.29 ; 95 \% \mathrm{CI}, 1.04-1.60$ and aRR, 1.33 ; 95\% CI, 1.04-1.69) (Table 3 ). Both the age of the woman and the extent of surgery (hysterectomy with bilateral salpingectomy vs hysterectomy) were individually significant risk factors for developing menopausal symptoms, but there was no significant interaction between the $2(P=.91)$. There was no additional risk for developing menopausal symptoms with higher age at the time of surgery and being subjected to opportunistic salpingectomy. In agestratified adjusted analysis, women at the age of 44-49 years remained at significantly increased risk of menopausal symptoms 1 year after bilateral salpingectomy (aRR, 1.53; 95\% CI, $1.06-2.20)$. In unadjusted analysis there was an increased risk even for women age $<40$ years (RR, 2.49; 95\% CI, 1.03-6.0), but after adjusting for potential confounders it was no longer significant (aRR, 2.29; 95\% CI, 0.80-6.48). However, the response rate to the menopause question in this age group was only $34 \%$. This is in part due to changes in the register, with cessation to include the question regarding menopausal symptoms to women $<40$ 
TABLE 1

Baseline patient characteristics for hysterectomy by laparoscopic or abdominal approach in 2013 through mid-2016

\begin{tabular}{|c|c|c|c|c|c|c|c|c|c|c|c|}
\hline \multirow[b]{2}{*}{ Characteristic } & \multicolumn{5}{|c|}{ Hysterectomy } & \multicolumn{5}{|c|}{ Hysterectomy with BSE } & \multirow[b]{2}{*}{$P$ value } \\
\hline & $\bar{n}$ & $\%$ & Mean \pm SD & Median & IQR $25-75$ & $\bar{n}$ & $\%$ & Mean \pm SD & Median & IQR $25-75$ & \\
\hline Age, $y$ & 3473 & & $44.1 \pm 6.1$ & 45 & $41-48$ & 1433 & & $45.1 \pm 5.2$ & 46 & $42-49$ & $<.001$ \\
\hline Body mass index & 2790 & & $26.4 \pm 4.7$ & 25.6 & $22.9-29.1$ & 1157 & & $26.5 \pm 4.5$ & 25.8 & $23.1-29.4$ & .45 \\
\hline Pregnancies & 2792 & & & 2.0 & $2.0-4.0$ & 1165 & & & 3.0 & $2.0-4.0$ & $<.001$ \\
\hline Parity & 2752 & & & 2.0 & $1.0-3.0$ & 1151 & & & 2.0 & $1.0-3.0$ & $<.001$ \\
\hline \multicolumn{12}{|l|}{ ASA classification } \\
\hline 1 & 2371 & 68.3 & & & & 934 & 66.2 & & & & \\
\hline 2 & 1056 & 40.4 & & & & 474 & 33.1 & & & & .08 \\
\hline 3 & 45 & 1.3 & & & & 25 & 1.7 & & & & \\
\hline Current smoking & 375 & 13.2 & & & & 143 & 12.1 & & & & .36 \\
\hline $\begin{array}{l}\text { Menopausal } \\
\text { symptoms, previous } \\
\text { or current }\end{array}$ & 423 & 23.5 & & & & 194 & 25.1 & & & & .36 \\
\hline $\begin{array}{l}\text { Previous abdominal } \\
\text { surgery }\end{array}$ & 1594 & 45.9 & & & & 631 & 44.0 & & & & .24 \\
\hline Endometriosis & 321 & 13.9 & & & & 136 & 14.4 & & & & .70 \\
\hline Previous salpingitis & 139 & 6.0 & & & & 77 & 8.1 & & & & .03 \\
\hline
\end{tabular}

years of age during the study period. The response rate in regard to menopausal symptoms postoperatively decreased with decreasing age in the women who had surgery 2013 through March 2015 and were included in the 1-year followup $(65.2 \%$ in women age $50-54$ years, $61.9 \%$ in women age $45-49$ years, $57.6 \%$ in women age $40-44$ years, and only $34.1 \%$ in women $<40$ years of age).

\section{Comment}

In this national retrospective registerbased clinical study, women going through hysterectomy with bilateral salpingectomy were at increased risk of reporting menopausal symptoms 1 year after surgery compared with women having hysterectomy only. Furthermore, there was a tendency toward higher risk of minor complications. The frequency of performing bilateral salpingectomy at the time of benign hysterectomy has increased in Sweden and indicates a general acceptance of the theory that opportunistic salpingectomy might prevent ovarian cancer, as well as the assumption that it can be done without harm.

A strength of our study is the large cohort and good coverage of the register, which has gradually increased the number of participating clinics to cover about $75 \%$ of clinics performing gynecological surgery in Sweden at the end of the study period. Thus, the results are likely to be applicable to a routine clinical setting in Sweden.

The patients reporting directly to the register, in combination with the physician's assessment, is a great strength in revealing the subjective symptoms in regard to menopause as well as a high detection rate of objective diagnoses. ${ }^{30,31}$ Furthermore, the follow-up rate is high with $83.5 \%$ responding at 8 weeks and $73.7 \%$ at 1 year.

A limitation is the retrospective design of the study with the inability to fully match the groups and adjust for all relevant factors. Therefore, residual as well as unmeasured confounding cannot be ruled out. The register changed strategy during the study period and stopped registering menopausal symptoms in women $<40$ years postoperatively, which makes it difficult to interpret the answers for the younger age groups.

There is increasing evidence that certain types of ovarian cancer originate in the fallopian tubes and opportunistic salpingectomy might be a feasible preventive measure, but there is still a lack of scientific support that this can be done in a safe manner without consequences for the woman. In our cohort there was a significantly higher risk of menopausal symptoms 1 year after being subjected to hysterectomy with bilateral salpingectomy in comparison with hysterectomy only. In the age-stratified analysis, the age group 45-49 years proved to be most at risk. It can be hypothesized that the ovaries in this premenopausal period are more vulnerable to damage from surgery in the surrounding tissue. It is also more likely that women in this age group present with menopausal symptoms within 1 year, in comparison to younger women, and we need a longer 
TABLE 2

Duration of surgery, perioperative bleeding, blood transfusion, hospital stay, days to normal activities of daily living, and days out of work after hysterectomy with bilateral salpingectomy compared with hysterectomy only, in multivariable analysis

\section{Duration of surgery}

Baseline duration of surgery

Hysterectomy with BSE vs hysterectomy

Abdominal vs laparoscopic approach

BMI, baseline 25

ASA 2 vs ASA 1

ASA 3 vs ASA 1

Age, baseline 30

Previous abdominal surgery

Endometriosis

Previous salpingitis

Perioperative bleeding*

Baseline perioperative bleeding

Hysterectomy with BSE vs hysterectomy

Abdominal vs laparoscopic approach

BMI, baseline 25

Current smoker vs nonsmoker

ASA 2 vs ASA 1

ASA 3 vs ASA 1

Previous salpingitis

Blood transfusion**

Baseline blood transfusion

Hysterectomy with BSE vs hysterectomy

Abdominal vs laparoscopic approach

BMI, baseline 25

Endometriosis

Hospital stay***

Baseline hospital stay

Hysterectomy with BSE vs hysterectomy

Abdominal vs laparoscopic approach

Duration of surgery, min

Endometriosis

ASA 2 vs ASA 1

ASA 3 vs ASA 1

Current smoker vs nonsmoker
Endometriosis

$$
\mathrm{n}=\mathbf{3 1 4 7}
$$

Min

114

$-2.7$

$-19.8$

$+1.4$

$+1.3$

$+4.8$

$+0.2$

$+0.8$

$+3.6$

$+3.6$

$\mathrm{n}=\mathbf{3 1 4 0}$

$\mathrm{mL}$

140

$-19.9$

$+117.7$

$(-39.8$ to -0.1$)$

$+8.7$

$+14.5$

$-56.6$

$+17.7$

$+48.2$

$-0.1$

U

$$
n=3122
$$

0.08

$-0.03$

(-0.8 to 0.02$)$

$+0.06$

(0.01 to 0.11 )

$+0.001$

$(-0.003$ to 0.006$)$

$-0.004$

Days

$n=3104$

0.7

$+0.1$

$+0.9$

$+0.006$

$(-0.07$ to 0.06$)$

(97.4 to 138)

(6.7 to 10.7)

(-12.3 to 41.4)

$(-83.8$ to -29.5$)$

$(-1.8$ to 37.2$)$

(-33.5 to 129.9)

(-36.9 to 36.7$)$

Collins et al. Complications after opportunistic salpingectomy. Am J Obstet Gynecol 2019.
$+0.2$

$+0.14$

$+0.38$

$-0.1$
$(0.02$ to 0.18$)$

(0.7 to 1.0$)$

(0.005 to 0.007$)$

(0.12 to 0.33$)$

(0.06 to 0.21$)$

$(0.06$ to 0.7$)$

$(-0.18$ to 0.03$)$ 


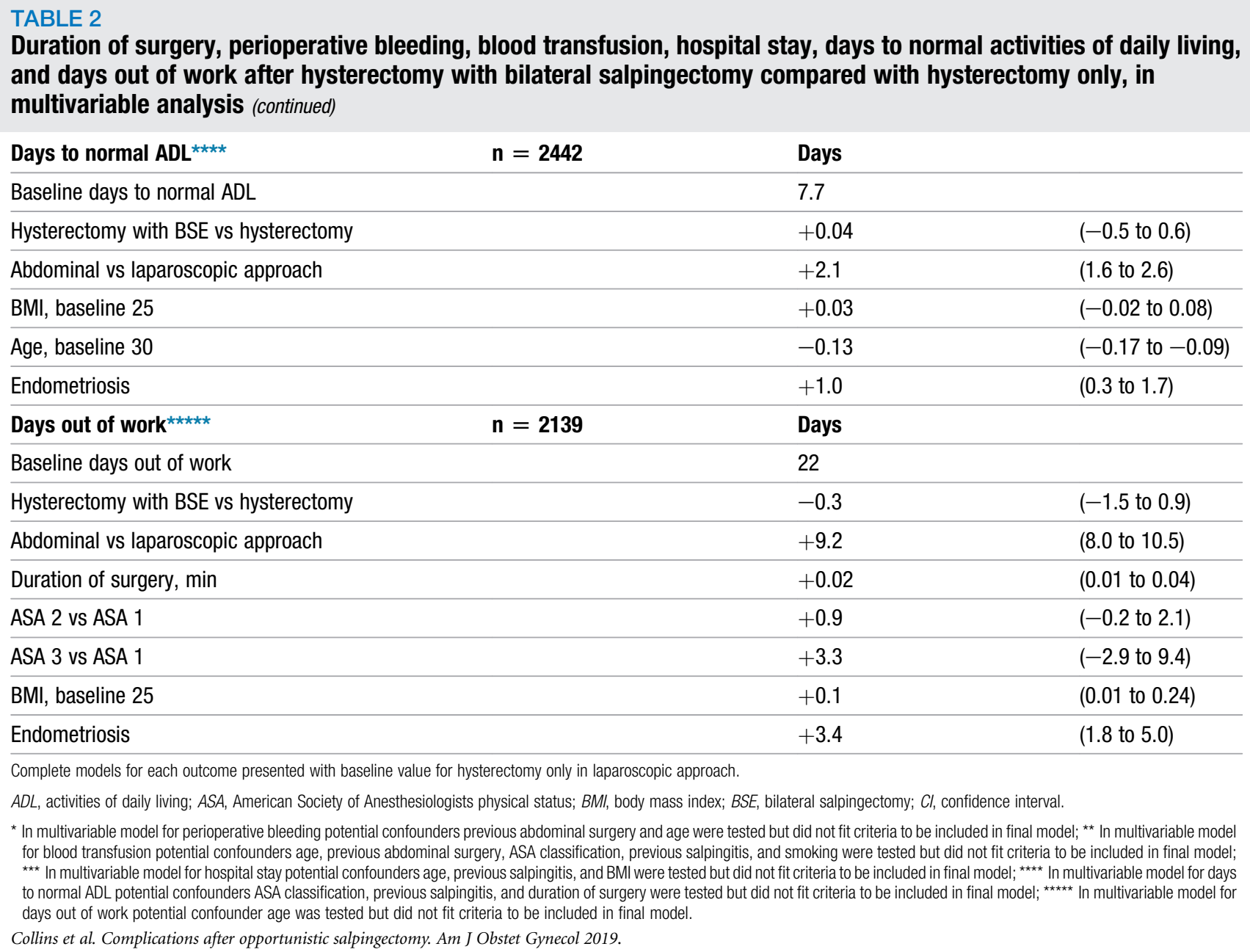

follow-up to assess the younger age groups. Mohamed et al suggested that a long-term decline in ovarian function could be secondary to chronic ischemia induced by salpingectomy, but evidence is insufficient. ${ }^{33}$ Sezik et al ${ }^{23}$ indicated a negative effect on ovarian blood supply 1 month after total salpingectomy compared with partial salpingectomy when performing hysterectomy in a small randomized trial. Chan et $\mathrm{al}^{34}$ revealed reduced blood flow in the ipsilateral ovary after laparoscopic salpingectomy performed due to ectopic pregnancy, supporting the theory of affected vascularity after surgery in the periovarian tissue.

It has been increasingly popular to use $\mathrm{AMH}$ to describe ovarian reserve, due to a small intracycle variability and its ability to predict time to menopause. ${ }^{35}$ $\mathrm{AMH}$ is validated for predicting age at natural menopause but loses accuracy in higher ages according to Depmann et $\mathrm{al}^{35}$ None of the studies focusing on AMH levels before and after opportunistic salpingectomy show any significant differences in comparison with hysterectomy only, ${ }^{15,20,22-24,26}$ but the longest follow-up is only 6 months in a study by Van Lieshout et al. ${ }^{26}$ The question is if $\mathrm{AMH}$, particularly with a short follow-up, is the accurate measure when assessing the long-term effects of opportunistic salpingectomy. In a meta-analysis by Mohamed et $\mathrm{al}^{33}$ focusing on AMH before and after bilateral salpingectomy, the authors comment on the short follow-up of existing studies and the small number of studies in the field, and suggest the results should be interpreted with caution. A normal AMH after surgery does not exclude the possibility of a more chronic ischemia with earlier development of menopause.

Our data revealed a tendency toward higher risk of minor complications up to 1 year after hysterectomy with bilateral salpingectomy. If we did not adjust for previous salpingitis, there was a significantly increased risk of minor complications 1 year after bilateral salpingectomy. Having a preexisting condition, ie, previous salpingitis, could be a reason to remove the fallopian tubes when performing a hysterectomy. Therefore, adding that variable to the adjusted model might obscure the effect of salpingectomy. Furthermore, the inclusion of previous salpingitis conferred a considerable loss of power due to a low response rate. Hence, it can be discussed which is the most accurate analysis and to retrieve reliable data we need studies in a randomized setting. 
TABLE 3

Menopausal symptoms and complications 1 year postoperatively in women having hysterectomy with bilateral salpingectomy or hysterectomy only

\begin{tabular}{|c|c|c|c|c|}
\hline & $\mathrm{n}$ & $\%, \% *$ & $\mathrm{RR}(\mathrm{Cl} 95 \%)$ & aRR $(95 \% \mathrm{Cl})$ \\
\hline \multicolumn{5}{|l|}{ Menopausal symptoms 1 y postoperatively } \\
\hline Hysterectomy with BSE vs hysterectomy & & $31.1,24.1$ & $1.29(1.04-1.60)$ & $1.33(1.04-1.69)$ \\
\hline Age, baseline 30 & & & $1.09(1.06-1.12)$ & $1.09(1.06-1.13)$ \\
\hline BMI, baseline 25 & & & $1.04(1.01-1.06)$ & $1.03(1.01-1.06)$ \\
\hline Current smoker vs nonsmoker & & & $1.55(1.19-2.02)$ & $1.45(1.09-1.93)$ \\
\hline ASA 2 vs ASA 1 & & & $1.28(1.06-1.56)$ & $1.16(0.93-1.46)$ \\
\hline Parity & & & $1.10(1.01-1.19)$ & $1.03(0.94-1.12)$ \\
\hline \multicolumn{5}{|l|}{ Hysterectomy with BSE vs hysterectomy ${ }^{* *}$} \\
\hline$<40 \mathrm{y}$ & $\mathrm{n}=148$ & & $2.49(1.03-6.00)$ & $2.29(0.80-6.48)$ \\
\hline $40-44$ y & $\mathrm{n}=371$ & & $1.16(0.68-1.96)$ & $0.93(0.48-1.76)$ \\
\hline $45-49$ y & $\mathrm{n}=659$ & & $1.37(0.99-1.89)$ & $1.53(1.06-2.20)$ \\
\hline $50-54$ y & $\mathrm{n}=305$ & & $1.15(0.80-1.66)$ & $1.17(0.78-1.75)$ \\
\hline Minor complications 1 y postoperatively *** & $n=1610$ & & & \\
\hline Hysterectomy with BSE vs hysterectomy & & $16.6,12.1$ & $1.36(1.05-1.77)$ & $1.30(0.93-1.83)$ \\
\hline Abdominal vs laparoscopic approach & & & $1.04(0.80-1.35)$ & $1.15(0.82-1.61)$ \\
\hline BMI, baseline 25 & & & $1.01(0.98-1.04)$ & $1.02(0.99-1.05)$ \\
\hline Current smoker vs nonsmoker & & & $1.32(0.93-1.86)$ & $1.15(0.75-1.74)$ \\
\hline Previous salpingitis & & & $1.64(1.02-2.64)$ & $1.60(0.99-2.59)$ \\
\hline Severe complications 1 y postoperatively ${ }^{\star \star \star \star}$ & $\mathbf{n}=\mathbf{1 8 9 0}$ & & & \\
\hline Hysterectomy with BSE vs hysterectomy & & $3.1,2.6$ & $1.20(0.67-2.14)$ & $1.08(0.51-2.27)$ \\
\hline Abdominal vs laparoscopic approach & & & $0.87(0.50-1.49)$ & $1.03(0.51-2.06)$ \\
\hline Total vs subtotal hysterectomy & & & $3.07(0.96-9.82)$ & $3.63(0.87-15.1)$ \\
\hline BMI, baseline 25 & & & $1.03(0.96-1.10)$ & $1.02(0.95-1.09)$ \\
\hline Age, baseline 30 & & & $0.94(0.90-0.98)$ & $0.94(0.88-0.99)$ \\
\hline \multicolumn{5}{|c|}{$A S A$, American Society of Anesthesiologists; $B M M$, body mass index; $B S E$, bilateral salpingectomy; $C l$, confidence interval; $R R$, relative risk; aRR, adjusted relative risk. } \\
\hline \multicolumn{5}{|c|}{ 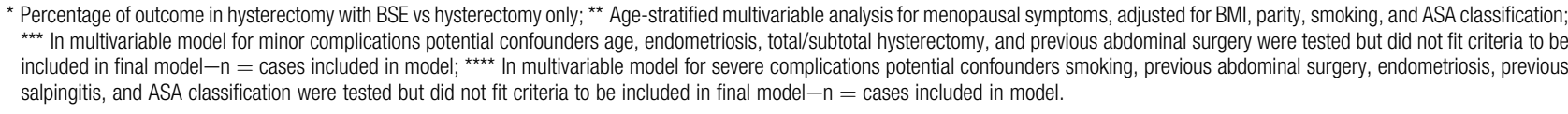 } \\
\hline \multicolumn{5}{|c|}{ Collins et al. Complications after opportunistic salpingectomy. Am J Obstet Gynecol 2019.} \\
\hline
\end{tabular}

The data on complications for each patient are cumulative, which explains why only the 1-year report finds significant differences. Our search did not identify any prior studies investigating complications to salpingectomy in this manner, with patient-reported complications and physician's assessment as a routine follow-up to surgery. The procedure has been successfully utilized regarding complications to hysterectomy in a recently published study. ${ }^{31}$ Studies reporting complications in a shorter time frame (0-6 months) have not shown any increased risk of complications. ${ }^{7,16,17,21,22,26}$ McAlpine et $\mathrm{al}^{6}$ conducted a retrospective cohort study reporting readmission as an indicator of complications and did not reveal any difference between the 2 groups. However, the overall quality of evidence in regards to complications was rated as very low in a recent systematic review by Darelius et al. ${ }^{13}$ The higher risk of ureter damage after salpingectomy found in this study has to be viewed with caution due to few cases, and the finding needs to be validated in future studies.
The results up to 8 weeks postsurgery showed a significant difference in hospital stay, with 0.1 day ( 2 hours and 24 minutes) extra after having had bilateral salpingectomy in addition to hysterectomy. Previous studies have not reported any difference in hospital stay ${ }^{8,17,26}$ and our results are, although significant, of uncertain clinical relevance. Perioperative bleeding was slightly reduced in the salpingectomy group and a similar result can be found in a retrospective study by Garcia et al. $^{8}$ The difference in the amount of blood loss was only $20 \mathrm{~mL}$ 
TABLE 4

Subgroups of complications reported 1 year after benign hysterectomy with bilateral salpingectomy vs hysterectomy only, abdominal or laparoscopic approach

\begin{tabular}{llccc}
$\begin{array}{l}\text { Location/type } \\
\text { of complication }\end{array}$ & Degree & $\begin{array}{l}\text { Hysterectomy, } \\
\mathbf{n}=1877\end{array}$ & $\begin{array}{c}\text { Hysterectomy with } \\
\text { BSE, } \mathbf{n}=477\end{array}$ & RR (95\% Cl) \\
\hline Ureter & Minor & $2(0.1 \%)$ & $2(0.4 \%)$ & $3,94(0.55-27.9)$ \\
\hline Intestines & Severe & $2(0.1 \%)$ & $5(1.0 \%)$ & $9.84(1.91-50.6)$ \\
\hline Bladder & Minor & $44(2.3 \%)$ & $9(1.9 \%)$ & $0.81(0.39-1.64)$ \\
\hline Abdominal wall & Severe & $8(0.4 \%)$ & $5(1.0 \%)$ & $2.46(0.80-7.49)$ \\
\hline & Minor & $63(3.4 \%)$ & $24(5.0 \%)$ & $1.50(0.95-2.38)$ \\
\hline Nerve & Severe & $16(0.9 \%)$ & $6(1.3 \%)$ & $1.48(0.58-3.76)$ \\
\hline & Minor & $76(4.0 \%)$ & $26(5.5 \%)$ & $1.35(0.87-2.08)$ \\
\hline Pain & Severe & $18(1.0 \%)$ & $7(1.5 \%)$ & $1.53(0.64-3.65)$ \\
\hline & Minor & $56(3.0 \%)$ & $20(4.2 \%)$ & $1.40(0.85-2.32)$ \\
\hline Vagina & Severe & $11(0.6 \%)$ & $4(0.8 \%)$ & $1.43(0.46-4.47)$ \\
\hline & Minor & $50(2.7 \%)$ & $18(3.8 \%)$ & $1.42(0.83-2.41)$ \\
\hline Fistula & Severe & $12(0.6 \%)$ & $3(0.6 \%)$ & $0.98(0.27-3.48)$ \\
\hline & Minor & $28(1.5 \%)$ & $12(2.5 \%)$ & $1.69(0.86-3.30)$ \\
\hline
\end{tabular}

$B S E$, bilateral salpingectomy; $C$, confidence interval; $R R$, relative risk.

Collins et al. Complications after opportunistic salpingectomy. Am J Obstet Gynecol 2019.

and, as expected, there was no difference in the need for blood transfusion. There were no differences in time in surgery, days to normal ADL, and days out of work, which corroborates the findings of several previous studies. ${ }^{6-8,16-18,20,26}$

In summary, bilateral salpingectomy at the time of hysterectomy was associated with an increased risk of menopausal symptoms and there was a tendency toward a higher risk of minor complications 1 year after surgery in this retrospective study. Randomized controlled trials with longer follow-up regarding ovarian function, surgical complications, as well as risk reduction of ovarian cancer are needed to correctly inform women on the risks and benefits of opportunistic salpingectomy.

\section{Acknowledgment}

We thank Mats Löfgren, Margareta Nilsson, and Birgitta Renström for support in handling the Swedish National Quality Register of Gynecological Surgery. All above-mentioned individuals are or have been employed by County Council of Västerbotten, Swedish National Quality Register of Gynecological Surgery.

\section{References}

1. Reid BM, Permuth JB, Sellers TA. Epidemiology of ovarian cancer: a review. Cancer Biol Med 2017;14:9-32.

2. Kurman RJ, Shih le M. Molecular pathogenesis and extraovarian origin of epithelial ovarian cancer-shifting the paradigm. Hum Pathol 2011;42:918-31.

3. Labidi-Galy SI, Papp E, Hallberg D, et al. High grade serous ovarian carcinomas originate in the fallopian tube. Nat Commun 2017;8:1093.

4. Falconer $H$, Yin L, Gronberg $H$, Altman D. Ovarian cancer risk after salpingectomy: a nationwide population-based study. J Natl Cancer Inst 2015;107.

5. Madsen C, Baandrup L, Dehlendorff C, Kjaer SK. Tubal ligation and salpingectomy and the risk of epithelial ovarian cancer and borderline ovarian tumors: a nationwide case-control study. Acta Obstet Gynecol Scand 2015;94: 86-94.

6. McAlpine JN, Hanley GE, Woo MM, et al. Opportunistic salpingectomy: uptake, risks, and complications of a regional initiative for ovarian cancer prevention. Am J Obstet Gynecol 2014;210:471.e1-11.

7. Till SR, Kobernik EK, Kamdar NS, et al. The use of opportunistic salpingectomy at the time of benign hysterectomy. J Minim Invasive Gynecol 2018:25:53-61.

8. Garcia C, Martin M, Tucker LY, et al. Experience with opportunistic salpingectomy in a large, community-based health system in the United States. Obstet Gynecol 2016;128: 277-83.

9. Perez-Lopez FR, Ceausu I, Depypere H, et al. Interventions to reduce the risk of ovarian and fallopian tube cancer: a European Menopause and Andropause Society position statement. Maturitas 2017;100:86-91.

10. Committee on Gynecologic Practice. Salpingectomy for ovarian cancer prevention. Committee opinion no. 620 [Erratum in College Publications (December 2015): Correction [Obstet Gynecol 2016] Obstet Gynecol 2016;127:405]. Obstet Gynecol 2015;125: 279-81.

11. Salvador S, Scott S, Francis JA, Agrawal A, Giede C. No. 344-Opportunistic salpingectomy and other methods of risk reduction for ovarian/ fallopian tube/peritoneal cancer in the general population. J Obstet Gynaecol Can 2017;39: 480-93. 
12. Chiaffarino $F$, Parazzini $F$, Decarli $A$, et al. Hysterectomy with or without unilateral oophorectomy and risk of ovarian cancer. Gynecol Oncol 2005;97:318-22.

13. Darelius A, Lycke M, Kindblom JM, Kristjansdottir B, Sundfeldt K, Strandell A. Efficacy of salpingectomy at hysterectomy to reduce the risk of epithelial ovarian cancer: a systematic review. BJOG 2017;124:880-9.

14. Kho RM, Wechter ME. Operative outcomes of opportunistic bilateral salpingectomy at the time of benign hysterectomy in low-risk premenopausal women: a systematic review. J Minim Invasive Gynecol 2017;24:218-29.

15. Findley AD, Siedhoff MT, Hobbs KA, et al. Short-term effects of salpingectomy during laparoscopic hysterectomy on ovarian reserve: a pilot randomized controlled trial. Fertil Steril 2013;100:1704-8.

16. Vorwergk J, Radosa MP, Nicolaus K, et al. Prophylactic bilateral salpingectomy (PBS) to reduce ovarian cancer risk incorporated in standard premenopausal hysterectomy: complications and re-operation rate. J Cancer Res Clin Oncol 2014;140:859-65.

17. Hanley GE, McAlpine JN, Pearce CL, Miller D. The performance and safety of bilateral salpingectomy for ovarian cancer prevention in the United States. Am J Obstet Gynecol 2017;216:270.e1-9.

18. Berlit S, Tuschy B, Kehl S, Brade J, Sutterlin M, Hornemann A. Laparoscopic supracervical hysterectomy with concomitant bilateral salpingectomy - why not? Anticancer Res 2013;33:2771-4.

19. Ghezzi F, Cromi A, Siesto G, Bergamini V, Zefiro F, Bolis P. Infectious morbidity after total laparoscopic hysterectomy: does concomitant salpingectomy make a difference? BJOG 2009;116:589-93.

20. Morelli M, Venturella $R$, Mocciaro $R$, et al. Prophylactic salpingectomy in premenopausal low-risk women for ovarian cancer: primum non nocere. Gynecol Oncol 2013;129: 448-51.

21. Minig L, Chuang L, Patrono MG, CardenasRebollo JM, Garcia-Donas J. Surgical outcomes and complications of prophylactic salpingec- tomy at the time of benign hysterectomy in premenopausal women. J Minim Invasive Gynecol 2015;22:653-7.

22. Song $T$, Kim MK, Kim ML, et al. Impact of opportunistic salpingectomy on anti-mullerian hormone in patients undergoing laparoscopic hysterectomy: a multicenter randomized controlled trial. BJOG 2017;124:314-20.

23. Sezik M, Ozkaya O, Demir F, Sezik HT, Kaya $\mathrm{H}$. Total salpingectomy during abdominal hysterectomy: effects on ovarian reserve and ovarian stromal blood flow. J Obstet Gynaecol Res 2007;33:863-9.

24. Tehranian A, Zangbar $\mathrm{RH}$, Aghajani $F$, Sepidarkish M, Rafiei S, Esfidani T. Effects of salpingectomy during abdominal hysterectomy on ovarian reserve: a randomized controlled trial. Gynecol Surg 2017;14:17.

25. Naaman $Y$, Hazan $Y$, Gillor $M$, et al. Does the addition of salpingectomy or fimbriectomy to hysterectomy in premenopausal patients compromise ovarian reserve? A prospective study. Eur J Obstet Gynecol Reprod Biol 2017;210:270-4.

26. Van Lieshout LAM, Pijlman B, Vos MC, et al. Opportunistic salpingectomy in women undergoing hysterectomy: results from the HYSTUB randomized controlled trial. Maturitas 2018;107: 1-6.

27. The National Quality Register of Gynecological Surgery. Available at: http://www2. gynop.se/home/about-gynop/. Accessed Nov. 8, 2018 .

28. Quality registries in Sweden. Available at: http://kvalitetsregister.se/englishpages/finda registry/registerarkivenglish/nationalqualityregistry forgynaecologicalsurgerygynop.2167.html. Accessed March 20, 2018.

29. Årsrapport Benign hysterektomi 2016. Available at: http://www2.gynop.se/wp-content/ uploads/2017/07/GynOp_\%C3\%85rsrapport_ Hysterektomi2016.pdf. Accessed Feb. 15, 2018.

30. Pakbaz M, Mogren I, Lofgren M. Outcomes of vaginal hysterectomy for uterovaginal prolapse: a population-based, retrospective, cross-sectional study of patient perceptions of results including sexual activity, urinary symptoms, and provided care. BMC Womens Health 2009;9:9.

31. Hesselman S, Hogberg U, Jonsson M. Effect of remote cesarean delivery on complications during hysterectomy: a cohort study. Am J Obstet Gynecol 2017;217:564.e1-8.

32. Hesselman S, Hogberg U, Rassjo EB, Schytt E, Lofgren M, Jonsson M. Abdominal adhesions in gynecologic surgery after cesarean section: a longitudinal population-based register study. BJOG 2018;125:597-603.

33. Mohamed AA, Yosef $A H$, James $C$, AlHussaini TK, Bedaiwy MA, Amer S. Ovarian reserve after salpingectomy: a systematic review and meta-analysis. Acta Obstet Gynecol Scand 2017;96:795-803.

34. Chan CC, Ng EH, Li CF, Ho PC. Impaired ovarian blood flow and reduced antral follicle count following laparoscopic salpingectomy for ectopic pregnancy. Hum Reprod 2003;18: 2175-80.

35. Depmann M, Eijkemans MJ, Broer SL, et al. Does anti-mullerian hormone predict menopause in the general population? Results of a prospective ongoing cohort study. Hum Reprod 2016;31:1579-87.

\section{Author and article information}

From the Department of Clinical Sciences, Obstetrics and Gynecology (Drs Collins and Idahl), and Epidemiology and Global Health Unit, Department of Public Health and Clinical Medicine (Mr Granåsen), Umeå University, Umeå; and Institute of Clinical Sciences, Department of Obstetrics and Gynecology, Sahlgrenska Academy, University of Gothenburg, Gothenburg (Dr Strandell), Sweden.

Received June 8, 2018; revised Sept. 26, 2018; accepted Oct. 8, 2018.

This work was supported by grants from the Cancer Research Foundation in Northern Sweden, grant number AMP 17-859. The funder was not involved in the collection, analysis, or interpretation of these data or in the decision to submit the article for publication.

The authors report no conflict of interest.

Presented at the 26th European Congress of the European Board and College of Obstetrics and Gynecology, Paris, France, March 8-10, 2018.

Corresponding author: Annika Idahl, MD, PhD. annika. idah|@umu.se 